\title{
AN INTERNAL CHARACTERIZATION OF THE PRIME RADICAL OF A JORDAN ALGEBRA
}

\author{
CHESTER TSAI
}

\begin{abstract}
The prime radical of a Jordan algebra $\mathfrak{N}$ is the set of all very strongly nilpotent elements of $\mathfrak{U}$.
\end{abstract}

Let $\mathfrak{i}$ be a quadratic Jordan algebra over a commutative, associative ring with 1. (For basic definitions, see [2].) Then an ideal $P$ of $\mathfrak{P}$ is a prime ideal of $\mathfrak{N}$ if, whenever $B$ and $C$ are ideals of $\mathfrak{2}$ and $B U_{C} \subseteq P$, then either $B \subseteq P$ or $C \subseteq P$. The prime radical of $\mathfrak{Q}$ is defined to be the intersection of all prime ideals of $\mathfrak{T}$. The concepts of prime ideal and prime radical of a linear Jordan algebra are discussed in [4]. One may apply these concepts to quadratic Jordan algebras without additional difficulty. (See [1].)

The treatment in [4] is incomplete as far as the internal structure of the prime radical of a Jordan algebra is concerned. One can show, among other things, that there exists a unique prime radical $P(\mathfrak{U})$ of a Jordan algebra $\mathfrak{A}$, which is the intersection of all prime ideals of $\mathfrak{A}$, and the prime radical of the quotient algebra $\mathfrak{U} / P(\mathfrak{U})$ is 0 . Moreover, if the radical of $\mathfrak{U}$ is zero then $\mathfrak{U}$ can be represented as a subdirect sum of prime algebras. However, very little was said about the prime radical itself besides that every element of $P(\mathfrak{U})$ is nilpotent, i.e., $P(\mathfrak{U})$ is a nil ideal of $\mathfrak{l}$.

For associative rings $\mathrm{J}$. Lambek gives an internal characterization of the prime radical $P(R)$ of a ring $R$. He shows, in [3], that an element $x$ of $R$ is in $P(R)$ if and only if $x$ is strongly nilpotent. In this paper we shall show a similar characterization for the prime radical of Jordan algebras.

Let $\mathscr{U}$ be a Jordan algebra over $\Phi$. If $a \in \mathfrak{A}$, then $[a]$ denotes the principal ideal of $\mathfrak{A}$ generated by $a$. A sequence $\left\{x_{0}, x_{1}, x_{2}, \cdots, x_{n}, \cdots\right\}$ of elements of $\mathfrak{R}$ will be called an $m$-sequence if $x_{n+1} \in J U_{x_{n}}$ and will be called an $M$ sequence if $x_{n+1} \in\left[x_{n}\right] U_{\left[x_{n}\right]}$. An element $x$ is called a strongly nilpotent element if every $m$-sequence beginning with $x_{0}=x$ is ultimately zero and is called very strongly nilpotent if every $M$-sequence beginning with $x_{0}=x$ is ultimately zero.

Received by the editors December 13, 1971 and, in revised form, February 10, 1972. AMS 1970 subject classifications. Primary 17C10.

Key words and phrases. Prime ideal, prime radical, strongly nilpotent element, very strongly nilpotent element, Jordan algebra.

(c) American Mathematical Society 1973 
LemMa 1. Every m-sequence in $\mathfrak{l}$ contains an $M$-subsequence.

Proof. Let $S=\left\{x_{0}, x_{1}, x_{2}, \cdots\right\}$ be an $m$-sequence of $\mathfrak{T}$. Then the subsequence $S^{\prime}=\left\{x_{0}^{\prime}, x_{1}^{\prime}, x_{2}^{\prime}, \cdots, x_{n}^{\prime}, \cdots\right\}$ where $x_{n}^{\prime}=x_{2 n}$ is a sequence of $\mathfrak{A}$. Since $S$ is an $m$-sequence for each $u$, there exists an element $y_{n} \in \mathfrak{N}$ such that $x_{n+1}=y_{n} U_{x_{n}}$. Thus for each $n$, we see $x_{n+1}^{\prime}=x_{2 n+2}=y_{2 n+1} U_{x_{2 n-1}}=$ $\left(y_{2 n+1} U_{x_{2 n}} U_{y_{2 n}}\right) U_{x_{2 n}}$ is an element of $\left[x_{n}^{\prime}\right] U_{\left[x_{n}^{\prime}\right]}$. Hence $S^{\prime}$ is an $M$ sequence. We see easily:

COROLLARY. Every very strongly nilpotent element of $\mathfrak{Q}$ is a strongly nilpotent element of $\mathfrak{H}$. Every strongly nilpotent element of $\mathfrak{N}$ is a nilpotent element.

THEOREM 1. The prime radical $P(\mathfrak{U})$ of $\mathfrak{U}$ is the set of all very strongly nilpotent elements in $\mathfrak{T}$.

Proof. Let $a$ be an element in $\mathfrak{A}$ but not in $P(\mathfrak{l l})$. Then there exists a prime ideal $P$ of $\mathfrak{A}$ which does not contain $a$. The set complement $P^{c}$ of $P$ is a $Q$-system (see [4]) which contains $a$. Let $a_{0}=a$ and $a_{1}$ be a nonzero element in $\left[a_{0}\right] U_{\left[a_{0}\right]} \cap P^{c}$ and, inductively, for each integer $n$ there exists a nonzero element $a_{n+1}$ in $\left[a_{n}\right] U_{\left[a_{n}\right]} \cap P^{c}$. Moreover, no member of the infinite sequence $S=\left\{a_{0}, a_{1}, a_{2}, \cdots\right\}$ can be zero so that $a$ is not a very strongly nilpotent element.

If $a$ is not a very strongly nilpotent element, then there exists an infinite sequence $T=\left\{a_{0}, a_{1}, a_{2}, \cdots\right\}, a_{0}=a, a_{n+1} \in\left[a_{n}\right] U_{\left[a_{n}\right]}, a_{k} \neq 0$. Let $P$ be a maximal ideal with respect to the property that is disjoint from $T$ (use Zorn's lemma). If one shows that $P$ is a prime ideal of 2 which misses $a$, then $a$ is not contained in the prime radical $P(\mathfrak{A})$.

Let $B$ and $D$ be ideals of $\mathfrak{U}$ not contained in $P$; then by the maximality of $P$ and the fact that both the ideals $B+P$ and $D+P$ properly contain $P$, there exist $a_{i}$ in $B+P$ and $a_{j}$ in $D+P$. For convenience, we let $i \leqq j$, then $a_{j} \in\left[a_{i}\right]$ and $\left[a_{j}\right] \subseteq\left[a_{i}\right]$ so that $a_{j+1} \in\left[a_{j}\right] U_{\left[a_{j}\right]} \subseteq\left[a_{j}\right] U_{\left[a_{i}\right]} \subseteq(D+P) U_{I j+l^{\prime}} \subseteq$ $D U_{B}+P$ and $a_{j+1} \notin P$. Hence $D U_{B} \nsubseteq P$, and $P$ is a prime ideal of $\mathfrak{X}$.

COROLLARY. If $K$ is an ideal of $\mathfrak{H}$, then the prime radical of $K$ consists of the set of all elements of $\mathfrak{Q}$ which are very strongly nilpotent mod $K$.

If $R$ is an associative algebra over $\Phi$ and $x$ is in $R$, then $x$ is strongly nilpotent if every sequence $\left\{x_{0}, x_{1}, x_{2}, \cdots\right\}$, such that $x_{0}=x$ and $x_{n+1}=$ $x_{n} r_{n} x_{n}$ for some $r_{n}$ in $R$, is ultimately zero. J. Lambek shows that the prime radical of $R$ is the set of strongly nilpotent elements of $R$.

It is well known that one may construct a quadratic Jordan algebra $R^{+}$on $R$ by defining the operator $U_{a}: y \rightarrow a y a$, cf. [2]. If $a$ is an element in $R$ (and so is in $R^{+}$), we shall say $a$ is strongly $R$-nilpotent if it is a strongly nilpotent element in $R$. Similarly we shall say $a$ is strongly (very strongly) $R^{+}$-nilpotent if it is a strongly (very strongly) nilpotent element in $R^{+}$. 
THEOREM 2. Let $R$ be an associative algebra over $\Phi$ and a be an element in $R$. Then the following statements are equivalent:

(1) $a$ is strongly R-nilpotent;

(2) a is strongly $R^{\dagger}$-nilpotent;

(3) $a$ is very strongly $R^{+}$-nilpotent.

Proof. We shall only show (1) implies (3), for the equivalence of (1) and (2) follows immediately from the definition. (3) implies (2) was shown by Lemma 1 and its corollary.

Since the prime radical $P(R)$ of $R$ is the set of all strongly $R$-nilpotent elements as pointed out by Lambek and the prime radical $P\left(R^{+}\right)$of $R^{+}$ is the set of all very strongly $R^{+}$-nilpotent elements in Theorem 1, we need only to show $P(R) \subseteq P\left(R^{+}\right)$.

Let $\Xi=\left\{K \mid K\right.$ is an ideal of $R$ contained in $\left.P\left(R^{+}\right)\right\}$. Then, by Zorn's lemma, there exists a maximal element $B$ in this collection. We note that $B$ is an ideal of $R$ and if $K$ is an ideal of $R$ such that $K^{2} \subseteq B$, then if one takes $K$ as an ideal of $R^{+}, K U_{K} \subseteq K^{2} \subseteq B \subseteq P\left(R^{+}\right)$. So $K \subseteq P\left(R^{+}\right)$. Thus, by the maximality of $B, K \subseteq B$. Therefore $B$ is a semiprime ideal of $R$ and $P(R) \subseteq B \subseteq P\left(R^{+}\right)$.

As a consequence of this argument we have a simple proof for a result of Erickson and Montgomery, [1, Theorem 4].

THEOREM 3 (ERICKSON AND MONTGOMERY). Let $R$ be an associative algebra over $\Phi$. Then the prime radical $P(R)$ of $R$ coincides with the prime radical $P\left(R^{+}\right)$of the Jordan algebra $R^{+}$.

Proof. Both $P(R)$ and $P\left(R^{+}\right)$consist of exactly the set of all strongly nilpotent elements of $R$ (or $R^{+}$).

We shall now consider an associative algebra $R$ with an involution $x \rightarrow x^{*}$. Consider that the space $S$ of ${ }^{*}$-symmetric elements is a quadratic Jordan algebra. We have

THEOREM 4. Let $R$ be an associative algebra with involution and a be an element in $S$, the space of symmetric elements. Then the following statements are equivalent:

(1) a is a strongly nilpotent element in $S$;

(2) $a$ is a very strongly nilpotent element in $S$;

(3) $a$ is very strongly $R^{\div}$-nilpotent;

(4) $a$ is strongly $R$-nilpotent.

We shall prove the following lemma first.

Lemma 2. If $a \in S$ and $a S a \subseteq P(R)$, then $a \in P(R)$.

Proof. For any $x, y \in R, a\left(x^{*} a x\right) a \in a S a \subseteq P(R)$ and $a\left(x a y+y^{*} a x^{*}\right) a \in$ $a S a \subseteq P(R)$. Thus $(a x a) y(a x a)=a\left(x a y+y^{*} a x^{*}\right) a x a-a y^{*}(a x * a x a) \in P(R)$. 
Hence $(a x a) R(a x a) \subseteq P(R)$ which yields axa $\in P(R)$ for $P(R)$ is a semiprime ideal in $R$. Now axa $\in P(R)$ for all $x \in R$ yields that $a R a \subseteq P(R)$ and so $a \in P(R)$ by the same reason.

ProOf OF THE THEOREM. We shall show (1) implies (4). If $a$ is not strongly $R$-nilpotent, then, by [1], $a \notin P(R)$. Let $a_{0}=a$, then, by Lemma 2 , $a_{0} S a_{0} \nsubseteq P(R)$. Thus there exists $s_{0} \in S$ such that $a_{1}=a_{0} s_{0} a_{0} \notin P(R)$. If $a_{n} \notin P(R)$ is obtained then there exists an element $s_{n}$ in $S$ so that $a_{n+1}=$ $a_{n} s_{n} a_{n} \notin P(R)$ by the same argument. Hence one finds an $m$-sequence $\left\{a_{0}, a_{1}, a_{2}, \cdots\right\}$ in $S$ which begins with $a$ and never terminates at zero. Therefore $a$ is not a strongly nilpotent element in the Jordan algebra $S$.

The proof of the theorem is essentially completed, for the implications (4) implies (3); (3) implies (2) and (2) implies (1) follow from the definition and Lemma 1 easily.

The following theorem of Erickson and Montgomery [1, Theorem 3] can now be proven in a very simple manner.

THEOREM 5 (ERICKSON AND MONTGOMERY). Let $R$ be an associative algebra with involution * and $S$ be the set of *-symmetric elements of $R$. Then the prime radical $P(S)$ of the Jordan algebra $S$ is the intersection $P(S)=S \cap P(R)$ of $S$ with the prime radical of $P(R)$ of the associative algebra $R$.

Proof. Both consist of the set of strongly nilpotent elements of $S$.

\section{REFERENCES}

1. T. S. Erickson and S. Montgomery, The prime radical in special Jordan rings, Trans. Amer. Math. Soc. 156 (1971), 155-164. MR 43 \#306.

2. N. Jacobson, Lecture note on quadratic Jordan algebra, Tata Institute, Bombay, India, 1969.

3. J. Lambek, Lectures on rings and modules, Blaisdell, Waltham, Mass., 1966, Chap. 3. MR 34 \#5857.

4. C. Tsai, The prime radical in a Jordan ring, Proc. Amer. Math. Soc. 19 (1968), 1171-1175. MR 37 \#6336.

Department of Mathematics, Michigan State University, East Lansing, MiCHIGAN 48823 